\title{
Simulação e análise econômica da produção in vivo e in vitro de embriões em bovinos
}

\author{
Renato Travassos Beltrame(1), Celia Raquel Quirino(2), Luis Gustavo Barioni(3) \\ e Vera Fernanda Martins Hossepian de Lima(4)
}

\begin{abstract}
(1)Centro Universitário do Espírito Santo, Rua Fioravante Ross, o 2.930, CEP 29703-900 Colatina, ES. E-mail: rtbeltrame@yahoo.com.br (2)Universidade Estadual do Norte Fluminense, Centro de Ciências e Tecnologias Agropecuárias, Avenida Alberto Lamego, no 2.000, CEP 28013-600 Campos dos Goytacazes, RJ. E-mail: crq@uenf.br ${ }^{(3)}$ Embrapa Informática Agropecuária, Caixa Postal 6041, CEP 13083-886 Campinas, SP. E-mail: barioni@cnptia.embrapa.br (4)Universidade Estadual Paulista, Faculdade de Ciências Agrárias e Veterinárias, Departamento de Medicina Veterinária Preventiva e Reprodução Animal, Via de Acesso Paulo Donato Castellane, s/no, Campus Jaboticabal, CEP 14884-900 Jaboticabal, SP. E-mail: veralima@fcav.unesp.br
\end{abstract}

Resumo - O objetivo deste trabalho foi avaliar o efeito das variáveis número de receptoras, protocolo de sincronização, indicadores de eficiência reprodutiva e custo da prenhez na eficiência econômica da produção in vivo e in vitro de embriões, em bovinos. Elaborou-se um aplicativo de simulação que permite ao usuário inserir os parâmetros das variáveis de entrada. Um cenário base, originado a partir de taxas tradicionais de eficiência das técnicas de produção in vivo (TE) e in vitro (PIVE) de embriões bovinos, foi introduzido no aplicativo de simulação como critério para comparação dos resultados. O aplicativo desenvolvido foi capaz de reproduzir os cenários de TE e PIVE. A produção de embriões foi simulada com o uso de simulação estocástica. Posteriormente determinou-se o número ótimo de receptoras por análise de sensibilidade. $\mathrm{O}$ valor presente líquido e o custo por prenhez foram utilizados como parâmetros de decisão. A sincronização para inovulação em tempo fixo reduziu a ociosidade de receptoras e, consequentemente, o custo final da prenhez em comparação à metodologia tradicional. A sexagem fetal deve estar associada à produção in vivo de embriões bovinos. Além disso, o número ótimo de receptoras por doadora é variável e dependente dos parâmetros de entrada do sistema.

Termos para indexação: Bos taurus, bovinos, modelos, receptoras, reprodução, viabilidade.

\section{Simulation and economic analysis of in vivo and in vitro bovine embryo production}

\begin{abstract}
The objective of this work was to evaluate the effect of the variables number of recipients, synchronization protocol, reproductive efficiency indicators and pregnancy cost, in the economic effectiveness of in vivo and in vitro bovine embryo production. A simulation application was elaborated to allow the user to insert the input variable parameters. A basic scenario, from the efficiency traditional rates of in vivo (ET) and in vitro production (IVP) techniques of bovine embryos, was introduced in the software as a criterion to compare the results. This software was able to reproduce both ET and IVP scenarios. The embryo production was simulated through stochastic simulation. The optimal number of recipients using sensitivity analysis was determined. The net present value and cost per pregnancy were used as a decision parameter. The synchronization for fixed-time embryo transfer decreased the recipient idleness and, consequently, the final cost of pregnancy, in comparison to the traditional methodology. Foetal sexing must be associated to IVP of bovine embryos. In addition, the optimal recipient number per donor is variable and depends on data inserted in the system.
\end{abstract}

Index terms: Bos taurus, cattle, models, recipients, reproduction, viability.

\section{Introdução}

Estudos econômicos das variáveis que influenciam os resultados das técnicas de produção in vivo (TE) e in vitro (PIVE) de embriões bovinos são escassos na literatura. A viabilidade dessas atividades depende dos índices reprodutivos, e é variável em diversas situações. Projeções antecipadas dos resultados econômicos da TE e PIVE facilitam a tomada de decisões na medida em que proporcionam a antecipação dos prováveis resultados e da formação do custo da prenhez.

Alguns trabalhos recentes têm tentado projetar a produção embrionária para uso em simulação. Em virtude de a variação na produção embrionária ser inerente ao processo reprodutivo, simulações e projetos que consideram o uso do número médio de embriões viáveis representam situações inexistentes e distantes da realidade. Essas situações têm sido minimizadas

Pesq. agropec. bras., Brasília, v.45, n.11, 1513-1520, dez. 2010 
quando metodologias corretas de simulação são implementadas (Beltrame et al., 2007, 2010; Beltrame, 2010).

Tanto na biotécnica de TE quanto na de PIVE, a grande variabilidade na produção de embriões pelas doadoras (Slenningl \& Wheeler, 1989; Galli et al., 2003) e a necessidade de compra e sincronização de receptoras, antes que se conheça o número de embriões coletados ou produzidos, dificultam a decisão acerca do número de animais que devem ser destinados à sincronização.

Com a finalidade de esclarecer esses problemas e projetar antecipadamente resultados de atividades que apresentem elevado risco, alguns modelos matemáticos têm sido desenvolvidos (Slenningl \& Wheeler, 1989; Ostergaard et al., 2005), para auxiliar na tomada de decisão em diferentes etapas do sistema de produção de bovinos. Entretanto, nenhum modelo foi direcionado a comparar e analisar aspectos econômicos e biológicos das biotécnicas de TE e PIVE.

O objetivo deste trabalho foi avaliar o efeito das variáveis número de receptoras, protocolos de sincronização, indicadores de eficiência reprodutiva e custo da prenhez na eficiência econômica da produção in vivo e in vitro de embriões, em bovinos.

\section{Material e Métodos}

Um modelo de simulação descrito previamente em Beltrame (2010) foi implementado no software de programação Delphi XE (Embarcadero Technologies, 2010), e permitiu ao usuário inserir parâmetros e índices técnicos e econômicos como variáveis de entrada.

Com o propósito de promover a visualização dos resultados da simulação, um cenário base originado em taxas tradicionais de desempenho reprodutivo nas técnicas de TE e PIVE, encontrados na bibliografia (Spell et al., 2001; Tomita, 2003; Barreiros et al., 2004) e obtidos no laboratório de Reprodução da Universidade Estadual Paulista, Jaboticabal, SP, foi proposto e introduzido no aplicativo de simulação como referência para comparações. Neste cenário, definiu-se que cinco doadoras seriam utilizadas em cada colheita e/ou aspiração, o período de simulação seria de dez anos, e o intervalo entre colheita ou aspiração seria de 60 dias para TE e 30 dias para PIVE.

As taxas e períodos utilizados no cenário base foram: intervalo protocolo por cio de 21 dias no TE; método de sincronização das receptoras à base de prostaglandina, que pressupôs a aplicação intervalada de 11 dias de fármaco à base de prostaglandina; a ciclicidade do rebanho de $70 \%$; taxa de sincronização das receptoras de $70 \%$; taxa de aptidão de $60 \%$, com receptoras prontas para inovulação; taxas de prenhez após a inovulação dos embriões a fresco (TE 50\% e PIVE $40 \%$ ) e descongelados (TE $30 \%$ e PIVE $25 \%$ ); e percentagem de machos e fêmeas oriundos de TE (50/50) e PIVE (57/43). Para a inserção dos custos mensais no modelo de simulação, foram utilizadas cinco categorias (adaptação - $\mathrm{R} \$ 62,60$; receptoras prontas para sincronizar - $\mathrm{R} \$ 34,20$; receptoras aptas - R\$ 56,20; aguardando diagnóstico de gestação $\mathrm{R} \$ 34,20$; após o diagnóstico de gestação - $\mathrm{R} \$ 38,50$ ), que englobaram as possibilidades de situação das receptoras na atividade.

O cálculo do item adaptação englobou os custos referentes à manutenção dos animais por um mês, e incluiu vermifugação, vacinação, medicamentos, honorários veterinários e exames (Tabela 1). A estimativa de custo das receptoras nas categorias prontas para sincronizar e que aguardavam diagnóstico foi relativa à manutenção mensal de cada receptora com custos estimados de pastagem ( $\mathrm{R} \$ 18,75$ ), mineralização ( $\mathrm{R}$ \$ 4,67), mão de obra $(\mathrm{R} \$ 10,27)$ e identificação/monitoramento ( $\mathrm{R} \$ 0,50)$, em um total mensal por receptora de $\mathrm{R} \$ 34,19$. Receptoras aptas englobam o somatório dos custos mensais de manutenção, o custo do protocolo de sincronização utilizado e, caso haja inovulação, antibioticoterapia.

Tabela 1. Estimativa de custos em reais das receptoras de embriões bovinos alocadas no modelo de simulação.

\begin{tabular}{lc}
\hline Item de custo & Total (R\$) \\
\hline Vermífugo (Ivermectina 1\%) & 1,50 \\
Vacinas (raiva, febre aftosa, clostridioses e leptospirose) & 9,70 \\
Mão de obra para observação de estro & 2,57 \\
Hormônios & \\
PGF $_{2 \alpha}{ }^{(1)}$ & 12,00 \\
$\mathrm{P}_{4}^{\left({ }^{(2)}\right.}$ & 38,40 \\
Exames (brucelose e tuberculose) $_{\text {Antibioticoterapia (aplicação por animal) }}$ & 13,00 \\
Avaliação reprodutiva (honorários veterinários por animal) & 10,00 \\
(1)PGF $_{2 \alpha}$, protocolo de sincronização do estro com base na aplicação \\
intramuscular de duas doses de prostaglandina com intervalo de 11 dias. \\
${ }^{(2)} \mathrm{P}_{4}$, protocolo de sincronização do estro e da ovulação com base em \\
implante intravaginal de progesterona (Nasser et al., 2004).
\end{tabular}


Nas estimativas, foram consideradas receptoras, mestiças adquiridas a um peso médio de $360 \mathrm{~kg}$ e um ágio de compra de $20 \%$. No descarte, assumiu-se um peso de $420 \mathrm{~kg}$. O preço de venda adotado foi calculado com base no preço caracterizado para o Estado de São Paulo, obtido para vaca gorda no mês de novembro de 2008 (Scot Consultoria, Nov, 2008). Neste caso, o preço de aquisição e de descarte de receptoras considerado foi de $\mathrm{R} \$ 1.008,00$ e $\mathrm{R} \$ 980,00$, respectivamente.

Referências financeiras mensais foram inseridas no modelo com base em cinco categorias conforme citado. Além desses custos, foram inseridos os campos para o custo de aquisição da doadora, valor residual da doadora, valor de descarte da receptora, custo de manutenção da doadora, custo do protocolo de superovulação ou aspiração, custo do embrião (PIVE), honorários para aspiração e valor de venda da prenhez não sexada, sexada, após ultrassonografia, de macho ou de fêmea (Tabela 2). Em razão da variabilidade entre potencial genético e custo de uma doadora de oócitos ou de embriões, convencionou-se neste trabalho a igualdade entre custo de aquisição e valor residual de doadoras.

O cenário foi proposto para contemplar a atividade em uma propriedade, onde taxas e custos tradicionais da TE e PIVE fossem introduzidos como dados de entrada. Assim, possibilitou-se a determinação da viabilidade dessa atividade na situação proposta. Nesse caso, custos de doadoras, receptoras e indicadores de eficiência reprodutiva foram completamente inseridos no modelo, e possibilitaram a determinação do valor presente líquido (VPL) e da taxa interna de retorno

Tabela 2. Valores considerados no cenário base em uma fazenda.

\begin{tabular}{lr}
\hline Item & Base (R\$) \\
\hline Custo de aquisição da doadora & $15.000,00$ \\
Valor residual da doadora & $15.000,00$ \\
Valor de descarte da receptora & 980,00 \\
Custo do protocolo de adaptação & 62,60 \\
Custo de manutenção da receptora & 34,20 \\
Custo de sincronização da receptora & 12,00 \\
Custo de manutenção da doadora & 49,19 \\
Custo do protocolo de superovulação & 303,20 \\
Honorários veterinários por doadora em: & \\
$\quad$ coleta & 500,00 \\
$\quad$ aspiração & 300,00 \\
Custo do embrião in vitro produzido & 70,00 \\
Valor de venda da prenhez & $2.500,00$ \\
Valor de venda da prenhez macho & $2.500,00$ \\
Valor de venda da prenhez fêmea & $4.000,00$ \\
\hline
\end{tabular}

(TIR), sob uma taxa de desconto de $0,5 \%$ mensal (Tabela 2).

No cenário base, o modelo foi utilizado para determinar e projetar um fluxo de caixa da atividade, de forma a estimar o número de prenhezes produzidas, as receitas provenientes do descarte de receptoras, da venda de prenhezes e os índices econômicos ao final do período. Uma relação de 40 receptoras por doadora na propriedade foi utilizada, tendo sido então utilizadas 200 receptoras em todos os cenários em um momento inicial.

Com a finalidade de observar a variação numérica provocada pela utilização de inovulação em tempo fixo (TETF), um cenário alternativo para TE $(\mathrm{C} 1=\mathrm{TE}$ TETF) e outro para PIVE (C2 = PIVE - TETF) foram projetados a partir dos cenários tradicionais (Tabela 3 ).

$\mathrm{Na}$ PIVE, a sexagem fetal também foi simulada em cenários adicionais. Nesse caso, considerouse a sexagem sendo realizada simultaneamente ao diagnóstico de gestação e ao custo de $\mathrm{R} \$ 70,00$ por procedimento. A cada inovulação de embriões provenientes de PIVE foram computados R \$20,00.

No caso da resolução por simulação, consideramse as ineficiências inerentes ao sistema. Uma vez que o número de embriões recuperados ou obtidos por intermédio de aspiração folicular é aleatório, o acréscimo no custo por prenhez pode ser diferente por causa da ociosidade de receptoras e da perda ou do congelamento de embriões. É necessário, portanto, definir um critério para estabelecer o ponto ótimo. Neste trabalho, decidiu-se que o critério seria o custo por prenhez e que o número ótimo de receptoras seria aquele no qual o custo por prenhez fosse mínimo para cada cenário e para cada biotécnica reprodutiva proposta.

As estimativas de custo inseridas no modelo tiveram como finalidade permitir o cálculo dos indicadores econômicos de rentabilidade VPL e a TIR (Puccini, 1999; Penedo, 2005), a determinação do número ótimo de receptoras, a comparação entre os protocolos de sincronização de receptoras e a escolha da técnica mais rentável nos cenários propostos. Essas estimativas tiveram como base valores de mercado pesquisados em novembro de 2008, e paridade de câmbio de US\$1,00 para $\mathrm{R} \$ 2,60$. A taxa de desconto utilizada foi de $0,5 \%$ ao mês. Neste caso, baseou-se em um negócio que obtivesse um rendimento de $6 \%$ ao ano, próximo ao oferecido pela caderneta de poupança. 
Alguns dos cenários considerados neste estudo dificilmente acontecerão na prática, mas foram necessários nessa parte experimental do trabalho. Assim, puderam-se avaliar algumas práticas no seu limite máximo de eficiência técnica e especulou-se sobre seu desempenho e resultados.

Como o objetivo do modelo é identificar se os ganhos propiciados pela tecnologia superam os custos adicionais que ela acarreta, é importante destacar que custos inerentes à atividade pecuária como instalações, manutenções e custos indiretos não foram considerados.

Para efeito de desenvolvimento do modelo e a fim de aproximar as estimativas de custo à realidade, assumiu-se que todas as doadoras foram coletadas consecutivamente por três vezes e/ou aspiradas durante período similar (seis meses), considerouse, então, a partir daí, uma gestação a termo. Foi considerado também um valor diferenciado de venda para as prenhezes após sexagem por ultrassonografia. É importante ressaltar que não se considerou correlação entre coletas para uma mesma doadora e, portanto, a geração de embriões foi realizada de forma estocástica, por meio da simulação de Monte Carlo, conforme Beltrame et al. $(2009,2010)$.

A compra e venda de doadoras embora tenha sido considerada biologicamente (substituição a cada seis meses) teve efeito financeiro nulo, por terem sido efetuadas as relações de compra e venda por preços idênticos e momentos similares da dinâmica temporal.

Em relação aos custos relativos a doadoras, valores similares foram utilizados na TE e PIVE para custos de aquisição, valor residual e manutenção destes animais. Estas informações estão apresentadas na Tabela 2. Mortalidade de animais, variáveis climáticas e alteração de variáveis nutricionais não foram consideradas, pois serão objeto de pesquisas futuras.

As simulações foram executadas 5.000 vezes para cada cenário, de modo a serem estabelecidas médias do número de prenhezes e índices de eficiência de cada cenário. Uma análise de sensibilidade do número de receptoras foi executada em cada caso na tentativa de identificar a relação ótima de uso de receptoras por doadora. Este número adequado de receptoras foi inserido no modelo para encontrar os coeficientes biológicos e financeiros que otimizavam a produção in vivo e in vitro de embriões. Esses novos cenários foram denominados Base-M e C1M para TE e Base-M e C2M para PIVE (cenários otimizados).

Estatisticamente utilizou-se a análise de variância do número de prenhezes para verificar se existiam diferenças entre os cenários simulados. As médias do número médio de prenhezes geradas foram comparadas pelo teste SNK (SAS Institute, 1999). Com base nesses resultados, uma extrapolação para a comparação monetária foi realizada.

Tabela 3. Cenários utilizados nas análises de simulação por técnicas de produção in vivo (TE) e in vitro (PIVE) em embriões bovinos.

\begin{tabular}{|c|c|c|c|c|}
\hline \multirow[t]{2}{*}{ Item } & \multicolumn{2}{|c|}{ Produção in vivo } & \multicolumn{2}{|c|}{ Produção in vitro } \\
\hline & Base & TETF $(\mathrm{C} 1)^{(1)}$ & Base & TETF $(\mathrm{C} 2)^{(2)}$ \\
\hline Período de simulação (anos) & 10 & 10 & 10 & 10 \\
\hline Intervalo entre coletas (dias) & 60 & 60 & 30 & 30 \\
\hline Adaptação (dias) & 50 & 50 & 50 & 50 \\
\hline Intervalo protocolo/cio (dias) & 14 & 10 & 14 & 10 \\
\hline Intervalo cio/TE (dia) & 7 & 7 & 7 & 7 \\
\hline Diagnóstico de gestação (dia) & 60 & 60 & 60 & 60 \\
\hline Protocolo & $\mathrm{PGF}_{2 \alpha}^{(3)}$ & $\mathrm{P}_{4}^{(4)}$ & $\mathrm{PGF}_{2 \alpha}$ & $\mathrm{P}_{4}$ \\
\hline Ciclicidade (\%) & 70,0 & 100,0 & 70,0 & 100,0 \\
\hline Sincronização (\%) & 70,0 & 100,0 & 70,0 & 100,0 \\
\hline Aptidão (\%) & 60,0 & 85,0 & 60,0 & 85,0 \\
\hline Concepção fresco (\%) & 50,0 & 50,0 & 40,0 & 40,0 \\
\hline Concepção descongelamento (\%) & 35,0 & 35,0 & 25,0 & 25,0 \\
\hline Estoque inicial de embriões & Nulo & Nulo & Nulo & Nulo \\
\hline Proporção macho:fêmea (\%:\%) & $50,0: 50,0$ & $50,0: 50,0$ & $43,0: 57,0$ & $43,0: 57$ \\
\hline
\end{tabular}

${ }^{(1)}$ Cenário alternativo para TE; (2) Cenário alternativo para PIVE; ${ }^{(3)} \mathrm{PGF}_{2 \alpha}$, protocolo de sincronização do estro com base na aplicação intramuscular de duas doses de prostaglandina com intervalos de 11 dias. ${ }^{(4)} \mathrm{P}_{4}$, protocolo de sincronização do estro e da ovulação com base em implante intravaginal de progesterona (Nasser et al., 2004). 


\section{Resultados e Discussão}

Todos os resultados apresentados foram obtidos com o programa de simulação desenvolvido, e destacam as principais variáveis físicas e econômicas para a realização da TE e PIVE em uma fazenda. Foi possível avaliar a migração do sistema de inovulação com observação de cio (tradicional) para um novo sistema (TETF). Na PIVE, a hipótese tradicional incorporou-se à sexagem fetal e sua posterior avaliação.

Da análise econômica, depreende-se a não utilização da TIR como parâmetro de decisão nos projetos com biotecnologia da reprodução associada à variabilidade das variáveis. Tal fato decorreu da impossibilidade de obter um valor absoluto para TIR e efetuar o processo de simulação pelo software Delphi XE (Embarcadero Technologies, 2010) em alguns dos cenários propostos. Assim, as comparações foram realizadas pelo VPL.

Quanto ao TE, foi possível identificar a inviabilidade dos projetos simulados, quando foi utilizado o número de 200 receptoras sugerido inicialmente (Tabela 4).
Ao análisar os cenários otimizados, percebeu-se a variabilidade do resultado nas situações propostas. Ao utilizar sêmen convencional, apenas o cenário com base na sincronização para inovulação em tempo fixo (C2M) apresentou viabilidade (Tabela 5).

Sugere-se que a sincronização para inovulação em tempo fixo, mesmo quando se consideram taxas de prenhez similares à sincronização tradicional, minimizou o número de receptoras na propriedade (95 animais), o que influenciou, de maneira positiva, o custo final da prenhez $(\mathrm{R} \$ 1.615,97)$.

A pequena diferença apresentada entre o custo da prenhez para o cenário base e a derivante otimizada dessa diferença é indicação de proximidade entre os dados de entrada sugeridos e impostos ao modelo (R\$1.846,53 x R\$ 1.844,12) na técnica de PIVE (Tabela 5).

No cenário base da TE, a análise de sensibilidade do VPL, em relação ao número de receptoras, é apresentada na Figura 1. Embora o VPL seja negativo em toda sua trajetória, identifica-se, pelo comportamento de sua

Tabela 4. Resultados do modelo de simulação para projeção da biotecnologia de produção in vivo de embriões bovinos em fazendas em um período de dez $\operatorname{anos}^{(1)}$.

\begin{tabular}{|c|c|c|c|c|}
\hline \multirow[t]{2}{*}{ Transferência de embriões } & \multicolumn{2}{|c|}{ Cenário base $-\mathrm{PGF}_{2 \alpha}$} & \multicolumn{2}{|c|}{ TETF $-\mathrm{P}_{4}$} \\
\hline & Base & Base $\mathrm{M}^{(2)}$ & $\mathrm{C} 1$ & $\mathrm{C} 1 \mathrm{M}^{(3)}$ \\
\hline Número de doadoras & 5 & 5 & 5 & 5 \\
\hline Número de receptoras & 200 & 160 & 200 & 95 \\
\hline Número de prenhezes & $1056 \mathrm{a}$ & $912 d$ & $957 b$ & $955 \mathrm{c}$ \\
\hline Custo médio por prenhez (R\$) & $1.935,67$ & $1.858,68$ & $4.531,10$ & $1.615,97$ \\
\hline Valor presente líquido - convencional (R\$) & $-106.729,49$ & $-16.310,45$ & $-2.558 .294,96$ & $212.862,90$ \\
\hline
\end{tabular}

Tabela 5. Resultados do modelo de simulação para projeção da biotecnologia de produção in vitro de embriões bovinos em fazendas em um período de dez anos ${ }^{(1)}$.

\begin{tabular}{|c|c|c|c|c|}
\hline \multirow[t]{2}{*}{$\overline{\mathrm{PIVE}}$} & \multicolumn{2}{|c|}{ Cenário base $-\mathrm{PGF}_{2 \alpha}$} & \multicolumn{2}{|c|}{ TETF $^{(2)}-\mathrm{P}_{4}$} \\
\hline & Base & Base $M^{(3)}$ & $\mathrm{C} 2$ & $\mathrm{C} 2 \mathrm{M}^{(4)}$ \\
\hline Número de doadoras & 5 & 5 & 5 & 5 \\
\hline Número de receptoras & 200 & 210 & 200 & 145 \\
\hline Número de fêmeas & $611 d$ & $636 \mathrm{c}$ & $793 a$ & $655 b$ \\
\hline \multirow[t]{2}{*}{ Número de prenhezes } & $1.421 \mathrm{~d}$ & $1.480 \mathrm{c}$ & $1.843 \mathrm{a}$ & $1.523 b$ \\
\hline & \multicolumn{4}{|c|}{ Sexagem convencional } \\
\hline Custo médio por prenhez $(\mathrm{R} \$)$ & $1.846,53$ & $1.844,12$ & $1.905,74$ & $1.719,79$ \\
\hline \multirow[t]{2}{*}{ Valor presente líquido (R\$) } & $-4.685,83$ & $-4.292,32$ & $-127.044,79$ & $184.566,36$ \\
\hline & \multicolumn{4}{|c|}{ Sexagem fetal } \\
\hline Custo médio por prenhez $(\mathrm{R} \$)$ & $1.897,84$ & $1.894,53$ & $1.952,41$ & $1.771,82$ \\
\hline Valor presente líquido (R\$) & $621.588,22$ & $612.746,56$ & $651.821,46$ & $776.875,78$ \\
\hline
\end{tabular}


curva, o número de receptoras que acarreta menor prejuízo no cenário simulado (32 receptoras por doadora utilizada). Assim, qualquer modificação no número total de prenhezes e no acréscimo ou decréscimo no número de receptoras ou doadoras não teria benefício econômico na atividade.

Para os cenários que envolvem PIVE, os indicadores exibem comportamento similar ao demonstrado na TE. Valores positivos são observados no cenário onde ocorre a sincronização para inovulação com tempo fixo (Tabela $5-\mathrm{C} 2 \mathrm{M}$ ).

A pressuposição da sexagem fetal na elaboração dos cenários tem como finalidade eliminar falhas decorrentes da taxa de acuidade em uma seleção de sexo e avaliar com precisão a venda de uma prenhez de um sexo característico. Entretanto, embora não esteja inserida na PIVE tradicional, sugere-se que a sexagem fetal esteja sempre associada a esses procedimentos, tendo em vista uma superioridade na proporção de machos obtidos (Rheingantz et al., 2004; Wolf et al., 2008). Nas projeções de longo prazo em que foi utilizada, com acréscimo de cerca de $3 \%$ ao valor de custo da prenhez, permitiu-se identificar o sexo do produto e agregar seu valor de venda (Tabela 5).

$\mathrm{Na}$ PIVE, ao realizar-se a análise dos indicadores econômicos, com a sexagem fetal por ultrassonografia no momento do diagnóstico de gestação, observou-se viabilidade econômica em todos os cenários simulados. Tal resultado pode ser decorrente de um montante de prenhezes similar àtécnica tradicional(cenário base-M), do diagnóstico do sexo precoce a baixo custo e do acréscimo no valor de venda em razão da pressuposta prenhez do sexo feminino. Grandes discrepâncias nos

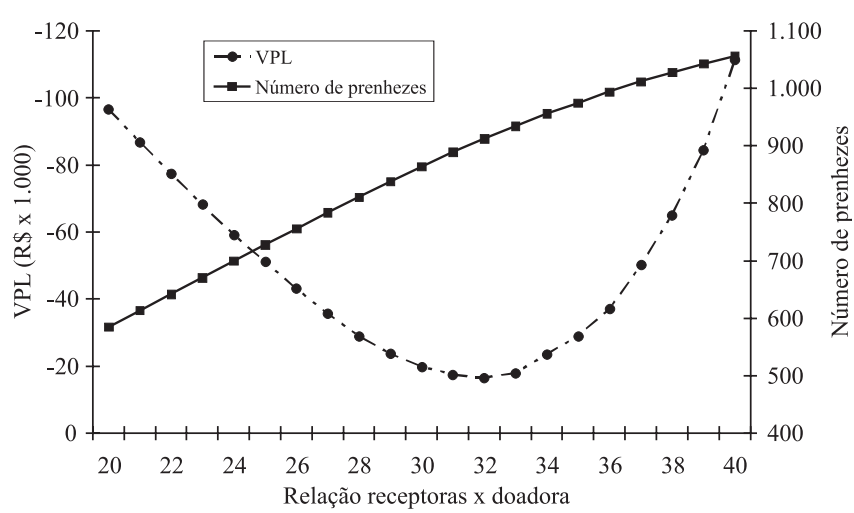

Figura 1. Valor Presente Líquido (VPL) e número de prenhezes com diferentes números de receptoras por doadora, no cenário base para produção in vivo de embriões bovinos. valores de VPL entre os cenários impostos e sugeridos foram proporcionais às diferenças entre o número de receptoras nas duas situações.

Nos cenários para TE e PIVE, foram obtidas menores estimativas para o custo da prenhez diante da sincronização de receptoras para inovulação em tempo fixo. Idéia similar já havia sido demonstrada em Beltrame et al. (2007), em inseminação artificial com sêmen convencional na TE.

Também na TE, diante da otimização dos cenários em que a inseminação artificial com sêmen convencional foi utilizada, o cenário otimizado (C1M), que considerou a sincronização de receptoras para inovulação em tempo fixo, possibilitou a produção de prenhezes ao custo de R\$ 1.615,97 cada uma (Tabela 4).

A determinação do número ótimo de receptoras pressupõe o custo mínimo da prenhez. Embora seja variável e individualizado nos diferentes sistemas, uma relação aparente entre as variáveis e um número ótimo de receptoras não foi definida. Entretanto, a redução da ociosidade no uso de receptoras, e o menor descarte de embriões foram considerados no modelo de simulação. Neste caso, o modelo foi capaz, em todos os cenários, de reproduzir uma situação real de escassez e de sobra de receptoras e de embriões, de forma a otimizar o número de receptoras que deveria ser utilizado para produzir prenhezes com custo mínimo (Tabelas 4 e 5).

Nos cenários em que a TETF foi considerada, um menor número de receptoras [(TE-TETF - 95) $\mathrm{x}$ (PIVE-TETF - 145)] foi necessário para otimização em relação ao protocolo tradicional. Adicionalmente, um custo inferior pôde ser obtido por prenhez diante da utilização desta metodologia de sincronização.

Em uma comparação entre as duas técnicas, observou-se que um montante maior de prenhezes foi obtido pela técnica de PIVE em todos os cenários (Tabela 6). Isto decorre de um menor intervalo entre aspirações e, consequentemente, uma maior produção de bezerros. Em relação à sincronização de receptoras, os cenários programados para TETF apresentaram menores estimativas de custo em comparação aos cenários que consideraram sincronização com prostaglandina.

Uma utilização conjunta de TE e PIV foi explorada por Pontes et al. (2009). Embora os procedimentos de PIVE tenham resultado em maior número de embriões produzidos (média de 9,4+5,3 da PIVE x 6,7+3,7 da $\mathrm{TE}$ ), os autores concluíram que a associação entre as 
Tabela 6. Comparação entre número de doadoras, receptoras, fêmeas, prenhezes e custo médio da prenhez (CMP) e valor presente líquido (VPL) para as técnicas de produção in vivo (TE) e in vitro (PIVE) de embriões bovinos nos cenários otimizados ${ }^{(1)}$.

\begin{tabular}{|c|c|c|c|c|}
\hline \multirow[t]{2}{*}{ Item } & \multicolumn{2}{|c|}{ Cenário base $-\mathrm{PGF}_{2 \alpha}$} & \multicolumn{2}{|c|}{ TETF $-\mathrm{P}_{4}$} \\
\hline & TE & PIVE & TE & PIVE \\
\hline Número de doadoras & 5 & 5 & 5 & 5 \\
\hline Número de receptoras & 160 & 210 & 95 & 145 \\
\hline Número de fêmeas & $456 \mathrm{~d}$ & $636 b$ & $477 \mathrm{c}$ & $655 \mathrm{a}$ \\
\hline Número de prenhezes & $912 d$ & $1480 \mathrm{~b}$ & $955 \mathrm{c}$ & $1.523 \mathrm{a}$ \\
\hline CMP (R\$) & $1.858,68$ & $1.844,12$ & $1.615,97$ & $1.719,79$ \\
\hline VPL - Convencional (R\$) & $-16.310,45$ & $-4.292,32$ & $212.862,90$ & $184.566,36$ \\
\hline
\end{tabular}

(1) Médias seguidas por letras iguais, nas linhas, não diferem entre si pelo teste de SNK, a 5\% de probabilidade.

técnicas é vantajosa. Entretanto, é interessante o estudo do efeito econômico do uso de receptoras neste sistema. Neste caso, ineficiências de uma biotécnica podem ser cobertas pelos benefícios da outra. Receptoras podem ter uma logística diferenciada de uso minimizando as despesas decorrentes de ociosidade.

Adisponibilidade de receptoras é um fator crucial no desenvolvimento de técnicas como TE e PIVE. Tendo em vista a escassez e as inconsistências inerentes à técnica, grande proporção dos custos é decorrente da variabilidade dos resultados. A utilização de protocolos de TETF pode minimizar a ociosidade de animais e a necessidade de observação de estro.

Destaca-se que a escolha pela biotécnica não determina a produção de prenhez com menor custo. Prenhez produzida pela técnica in vivo pode custar menos em comparação aos produtos produzidos por PIVE, ou vice-versa. A interação entre os dados de entrada nos cenários e a utilização do número ótimo de receptoras por doadora são fundamentais na determinação do resultado final e da técnica a utilizar.

É importante ressaltar que os indicadores econômicos apresentados refletem a viabilidade das técnicas estudadas nos cenários elaborados, e não da atividade, já que não foram considerados todos os custos envolvidos. Os indicadores econômicos foram calculados em relação ao momento, mas oscilações podem ocorrer.

\section{Conclusões}

1. Nas técnicas de produção in vivo e in vitro de embriões bovinos o número de receptoras e o protocolo de sincronização utilizado influenciam diretamente o custo final da prenhez.

2. O uso do número ótimo de receptoras por doadora determina a produção de prenhezes com menor custo e, consequentemente, a escolha pela técnica a se utilizar.

\section{Agradecimentos}

Ao Conselho Nacional de Desenvolvimento Científico e Tecnológico, pela bolsa de doutorado ao primeiro autor.

\section{Referências}

BARREIROS, T.R.R.; BLASCHI, W.; BORSATO, E.A.; LUDWIG, H.E.; MEIRA DA SILVA, D.R.; SENEDA, M.M. Comparação das taxas de prenhez entre receptoras com corpos lúteos cavitários ou compactos após protocolo de sincronização com cloprostenol ou transferência de embriões em tempo fixo. Semina: Ciências Agrárias, v.27, p.657-664, 2006.

BELTRAME, R.T. Impacto bioeconômico da sexagem de espermatozóides em programas de transferência de embriões e fertilização in vitro em zebuínos. 2010. 113p. Tese (Doutorado) - Universidade Estadual do Norte Fluminense, Campos dos Goytacazes.

BELTRAME, R.T.; BARIONI, L.G.; MAESTRI, B.D.; QUIRINO, C.R. Economic optimization of the number of recipients in bovine embryo transfer programs. Scientia Agricola, v.64, p.221-226, 2007.

BELTRAME, R.T.; BARIONI, L.G.; QUIRINO, C.R.; DANTAS, O.D. Probability density function of the number of Embryos collected from superovulated Nelore breed donors. Scientia Agricola, v.66, p.447-450, 2009.

BELTRAME, R.T.; QUIRINO, C.R.; BARIONI, L.G.; DIAS A.J.B.; SOUZA, P.M. Análise da produção de embriões na fertilização in vitro e transferência de embriões para doadoras Nelore. Ciência Animal Brasileira, v.11, p.21-27, 2010.

EMBARCADERO TECHNOLOGIES. Delphi XE. San Francisco, California, 2010. Available at: <http://www.embarcadero.com/br/ products/delphi>. Accessed on: 20 dez. 2010.

GALLI, C.; DUCHI, R.; CROTTI, G.; TURINI, P. Bovine embryo technologies. Theriogenology, v.59, p.599-616, 2003.

NASSER, L.F.; REIS, E.L.; OLIVEIRA, M A.; BÓ, G.A.; BARUSELLI P.S. Comparison of four synchronization protocols for fixed-time bovine embryo transfer in Bos indicus x Bos taurus recipients. Theriogenology, v.62, p.1577-1584, 2004. 
OSTERGAARD, S.; FRIGGENS, N.C.; CHAGUNDA, M.G.G. Technical and economic effects of an inline progesterone indicator in a dairy herd estimated by stochastic simulation. Theriogenology, v.64, p.819-843, 2005.

PENEDO, R.C. A taxa interna de retorno na análise de investimentos. Brasília: Lettera, 2005. 371p.

PONTES, J.H.F.; NONATO-JUNIOR, I.; SANCHES, B.V.; ERENO-JUNIOR, J. C.; UVO, S.; BARREIROS, T. R. R.; OLIVEIRA, J.A.; HASLER, J.F.; SENEDA, M.M. Comparison of embryo yield and pregnancy rate between in vivo and in vitro methods in the same Nelore (Bos indicus) donor cows. Theriogenology, v.71, p.690-697, 2009.

PUCCINI, A.L. Matemática financeira objetiva e aplicada. 6.ed. São Paulo: Saraiva, 1999. 440p.

RHEINGANTZ, M.G.T.; PEGORARO, L.M.C.; DELLAGOSTIN, O.A.; PIMENTEL, A.M.; BERNARDI, M.L.; DESCHAMPS, J.C. Proporção macho: fêmea de embriões bovinos cultivados na presença ou ausência de glicose após FIV com espermatozóides selecionados por Swim-up ou Gradiente de Percoll. Brazilian
Journal of Veterinary Research and Animal Science, v.41, p.32-39, 2004.

SAS INSTITUTE. SAS/STAT: user's guide statistics. Version 9.2. Cary: SAS Institute, 1999.

SLENNINGL, B.D.; WHEELER, M.B. Risk evaluation for bovine embryo transfer services using computer simulation and economic decision theory. Theriogenology, v.31, p.653-673, 1989.

SPELL,A.R.; BEAL, W.E.; CORHA, L.R.; LAMB, C.G. Evaluating recipient and embryo factors that affect pregnancy rates of embryo transfer in beef cattle. Theriogenology, v.56, p.287-297, 2001.

TOMITA, S.Y. Viabilidade prática e custo da indução de gestações gemelares em bovinos de corte, a partir de embriões produzidos in vitro e criopreservados para transferência direta. 2003. 70p. Tese (Doutorado) - Universidade Estadual Paulista, Jaboticabal.

WOLF, C.A.; BRASS, K.E.; RUBIN, M.I.B.; POZZOBON, S.E.; MOZZAQUATRO, F.D.; DE LA CORTE, F.D. The effect of sperm selection by Percoll or swim-up on the sex ratio of in vitro produced bovine embryos. Animal Reproduction, v.5, p.110-115, 2008.

Recebido em 30 de agosto de 2010 e aprovado em 8 de outubro de 2010 